\title{
Biomarkers of myocardial injury with different energy sources for atrial fibrillation catheter ablation
}

\author{
Michela Casella ${ }^{1}$, Antonio Dello Russo ${ }^{1}$, Eleonora Russo ${ }^{1}$, Ghaliah Al-Mohani ${ }^{1}$, \\ Pasquale Santangeli ${ }^{2}$, Stefania Riva ${ }^{1}$, Gaetano Fassini ${ }^{1}$, Massimo Moltrasio ${ }^{1}$, \\ Ester Innocenti ${ }^{1}$, Daniele Colombo ${ }^{1}$, Fabrizio Bologna ${ }^{1}$, Gennaro Izzo ${ }^{1}$, \\ Joseph G. Gallinghouse ${ }^{2}$, Luigi Di Biase ${ }^{2}$, Andrea Natale ${ }^{2}$, Claudio Tondo ${ }^{1}$ \\ ${ }^{1}$ Cardiac Arrhythmia Research Centre, Centro Cardiologico Monzino IRCCS, Milan, Italy \\ ${ }^{2}$ Texas Cardiac Arrhythmia Institute at St. Davis Medical Center, Austin, Texas, USA
}

\begin{abstract}
Background: Our study aims to compare acute myocardial injury biomarker rise after atrial fibrillation ablation performed with different technologies.

Methods and Results: One hundred and ten patients were treated with pulmonary vein isolation with 4 different technologies: open-irrigated tip radiofrequency $(R F)$ catheter in 35 patients (Group A), cryoballoon in 35 patients (Group B), visually guided laser balloon in 20 patients (Group C), open-irrigated tip $R F$ catheter with contact-force-sensing technology in 20 patients (Group D). Post-procedure samples of cardiac troponin I (cTnI) and creatinine kinase$-M B(C K-M B)$ were collected at $19 \pm 3 \mathrm{~h}$ and $43 \pm 3 \mathrm{~h}$ after ablation. At the first postprocedural sample, cTnI and CK-MB levels were found elevated in all 110 patients with a median value of $2.11 \mathrm{ng} / \mathrm{mL}$ and $8.95 \mathrm{ng} / \mathrm{mL}$, respectively. Group B showed cTnI levels increased (median $5.96 \mathrm{ng} / \mathrm{mL}$ ) compared to other groups (median Group A: $1.72 \mathrm{ng} / \mathrm{mL}$, Group C: $1.54 \mathrm{ng} / \mathrm{mL}$, Group D: $2.0 \mathrm{ng} / \mathrm{mL} ; p<0.001$ ). Also CK-MB levels resulted higher in cryoablation (median $26.4 \mathrm{ng} / \mathrm{mL}$ ) compared to other groups (median Group A: $6.40 \mathrm{ng} / \mathrm{mL}$, Group C: $7.15 \mathrm{ng} / \mathrm{mL}$, Group D: $6.50 \mathrm{ng} / \mathrm{mL} ; p<0.001$ ). No significant association was observed between biomarker levels and recurrences of atrial fibrillation after a mean follow-up of $369 \pm 196$ days.
\end{abstract}

Conclusions: Highest markers for myocardial injury were observed in the cryoballoon group. It is possible that a longer delivery energy duration and other factors affecting lesion size resulted in higher amount of cardiac injury in cryoablation. The higher levels of cardiac biomarkers did not translate into a better outcome and its physiologic significance is unknown. (Cardiol J 2014; 21, 5: 516-523)

Key words: cardiac biochemical markers, cardiac troponin I, creatinine kinase-MB, atrial fibrillation ablation, myocardial injury

\section{Introduction}

Pulmonary vein $(\mathrm{PV})$ isolation has become the mainstay of interventional treatment of paroxysmal atrial fibrillation (AF) using catheter ablation [1]. Nevertheless, recovery of conduction between the PVs and the left atrium is relatively common after a successful initial procedure and it is strictly related to arrhythmia recurrence [2]. Permanent lesion creation has been the target of new technological improvements in ablation catheters. Initially the catheter tip irrigation allowed

Address for correspondence: Eleonora Russo, MD, Cardiac Arrhythmia Research Centre, Centro Cardiologico Monzino IRCCS, Via Parea 4, 20138 Milan, Italy, tel: 003902 58002340, fax: 003902 58002398, e-mail: elerusso81@hotmail.it 
to perform more extensive lesions; more recently these catheters have been strengthened with the assistance of contact-force-sensing technology to ensure a real-time, objective measure of contact force between the catheter tip and the beating heart wall during ablation. Furthermore, durability of lesion can be ameliorated by the utilization of new ablation catheter configurations, for example balloon catheters, or by the utilization of alternative sources of energy, such as cryothermal and laser energies [3, 4].

As several markers have been proven to be useful for diagnosis and evaluation of size of myocardial injury after acute ischemic episode, many authors tried to implement different biomarkers to evaluate and quantify the size of effective ablation lesions [5-18]. On one hand, previous studies have univocally shown humoral biomarkers for myocardial injury to be elevated after radiofrequency (RF) ablation, depending on the amount of energy delivered and the site of ablation [5]. On the other hand, contrasting data exist about myocardial injury biomarker trend after cryoablation procedures [6-9]. Furthermore, to date, no data have been available on these biomarkers after laser balloon ablation or after RF catheter ablation with the assistance of contact-force-sensing technology. In addition, it is unknown whether myocardial injury biomarker could predict persistent lesion at long-term follow-up and which is its role with regards to arrhythmia recurrence.

The purpose of this study was to compare cardiac troponin I (cTnI) and creatinine kinase isoenzyme $\mathrm{MB}$ (CK-MB) rise after AF ablation performed with 1 of 4 different technologies: conventional irrigated tip RF catheter, cryoballoon, visually guided laser balloon, irrigated tip $\mathrm{RF}$ catheter with contact-force-sensing technology. In addition, the biomarker rise was associated to clinical outcomes during follow-up.

\section{Methods}

\section{Study population}

We prospectively enrolled 110 consecutive patients suffering from drug refractory paroxysmal $\mathrm{AF}$ and admitted to our institution to perform catheter ablation. Paroxysmal AF was defined as episodes which converted spontaneously to sinus rhythm within 7 days or were sometimes terminated by electrical or pharmacological cardioversion within $48 \mathrm{~h}$. Patients were randomized to PV isolation with either conventional open-irrigated tip RF catheter (35 patients, Group A) or with cryoballoon catheter (35 patients, Group B), or with visually guided laser balloon (20 patients, Group C), and open-irrigated tip RF catheter with contact-force-sensing technology (20 patients, Group D). Only patients with normal baseline values for myocardial injury were included. We excluded patients with renal failure, myopathy, recent myocardial ischemia or clinical signs of infection.

\section{Ablation procedure}

Informed consent was obtained from all patients. Transseptal puncture was performed under fluoroscopic guidance only or with the adjunct of intracardiac echocardiography at physician's discretion. After transseptal puncture, intravenous heparin was used to maintain an activated clotting time of $300-400 \mathrm{~s}$. In all procedures, an esophageal temperature probe was used to monitor esophageal temperature changes during energy delivery. Energy delivery duration was also calculated and recorded.

\section{Conventional open-irrigated tip RF catheter ablation}

A single or double transseptal puncture was performed at physician's discretion. For RF ablation, an open irrigated-tip catheter with a $3.5-\mathrm{mm}$ -tip electrode (ThermoCool, Biosense Webster) was used in conjunction with a 3-dimensional electroanatomic mapping system (CARTO 3, Biosense Webster or NavX Fusion, St. Jude Medical). $\mathrm{RF}$ energy was delivered with power of up to $35 \mathrm{~W}$ and a maximum temperature of $43^{\circ} \mathrm{C}$. Ablation was performed circumferentially, guided by a circular mapping catheter, near the antrum of each PV. The procedural endpoint was $\mathrm{PV}$ isolation as confirmed by the circumferential mapping catheter and pacing maneuvers (Lasso, Biosense Webster, Diamond Bar, CA, USA).

\section{Cryoballoon ablation}

A single or double transseptal puncture was performed according to the use of a conventional circumferential mapping catheter or the customized mapping catheter Achieve (Medtronic, Minneapolis, MN, USA). Positioning of the $28-\mathrm{mm}$ or 23-mm diameter cryoballoon (Artic Front Medtronic, Minneapolis, MN, USA) was achieved using the guidewire and the $12.5 \mathrm{Fr}$-steerable sheath (Flexcath Medtronic Minneapolis, MN, USA). Only in 5 patients we adopted a second generation mapping catheter (Arctic Front Advance Cardiac CryoAblation Catheter System Medtronic, Minneapolis, MN, USA). 
Because of a risk of right phrenic nerve palsy, while delivering cryoenergy to right PVs, a $6 \mathrm{~F}$ quadripolar catheter was positioned in the superior vena cava for phrenic nerve stimulation. After confirmation of PV occlusion by contrast injection, the 5-min freezing cycle was initiated (4-min with Arctic Front Advance). After 2 freezing cycles, PV isolation was assessed by circumferential mapping catheter.

\section{Visually guided laser balloon ablation}

Two transseptal punctures were performed for the ablation catheter and multipolar mapping catheter. After the dedicated 15 Fr deflectable sheath (Cardiofocus Inc.) was placed in the left atrium, the balloon catheter was inserted, inflated within the atrium, and sequentially positioned at each PV ostium. Energy delivery was performed with a power of 8.5-10 Watt for $20 \mathrm{~s}$. Before delivery of energy to the right PVs, a pacing catheter was positioned within the superior vena cava for phrenic nerve stimulation. A Lasso catheter was used to interrogate each PV before and after ablation to assess whether electric isolation was achieved.

\section{Open-irrigated tip RF catheter ablation with contact-force-sensing technology}

Patients' instrumentation was performed as in conventional procedures. All ablations were performed with a contact force sensing catheter (Tacticath $^{\mathrm{TM}}$ Endosense SA, or Contact Therapy ${ }^{\mathrm{TM}}$ Cool Path ${ }^{\mathrm{TM}}$ St. Jude Medical), which is a $7 \mathrm{Fr}$, open irrigated-tip catheter. The ablation catheter was connected through the splitter unit simultaneously to the RF generator and the force sensing base system. Energy delivery duration, mean contact force and force time integral (FTI) were also recorded and calculated.

\section{Blood sampling and Biomarker measurements}

Baseline blood tests were drawn at the time of hospital admission the day before the scheduled procedure in all patients (cTnI and CK-MB). Post-procedure samples were collected in the following 2 mornings after ablation.

Serum samples were processed at our hospital's central laboratory facility. Within $30 \mathrm{~min}$ of collection, samples were centrifuged. cTnI was measured with the commercially available Access ${ }^{\circledR}$ AccuTnI ${ }^{\mathrm{TM}}$ assay (Beckman Coulter, Chaska, MN), CK-MB were tested by separate enzymatic commercially available test kits (CK-MB test). CK-MB and cTnI cut-off values ( $\geq 6.5 \mathrm{ng} / \mathrm{mL}, \geq 0.1 \mathrm{ng} / \mathrm{mL}$, respectively) were treated as pathologically increased.

\section{Postablation management and follow-up}

Regular follow-up consisted of outpatient clinic visits at $1,3,6$, and 12 months after the procedure and included a detailed history, physical examination, 12-lead electrocardiogram, 24-h or 7-day Holter monitoring. Recurrence of AF post ablation is defined as a recurrence of AF more than 3 months following AF ablation. Episodes of atrial tachycardia or atrial flutter should also be classified as a "recurrence". Recurrence rates were analyzed with a postprocedural blanking period of 3 months.

\section{Statistical analysis}

Data are reported as mean \pm standard deviation. The distribution of variables was assessed by Kolmogorov-Smirnov test. Categorical variables, presented as counts and percentages, were compared in cross-tabulation tables by means of the Pearson $\chi^{2}$ test. The non-parametric Kruskal-Wallis and Wilkoxon tests were used to investigate differences among groups. Correlations of serum cTnI and CK-MB levels with procedural variables were performed using linear regression analysis (Pearson). These data were analyzed by the SPSS 20.0 statistical software (SPSS Italia, Inc., Florence, Italy). The threshold of statistical significance was set at a $\mathrm{p}$ value of $<0.05$.

\section{Results}

\section{Clinical characteristics}

The study population consisted of 110 consecutive patients referred for ablation of drug resistant $\mathrm{AF}$ (76\% male, mean age $57.2 \pm 11.3$ years). Patients baseline characteristics did not differ among groups (Table 1).

\section{Procedural data}

The procedural endpoint of complete PV isolation was achieved in all 110 patients. In Group A patients, all PVs were successfully isolated with a mean of $27 \pm 16$ pulses with $R F$ and a mean energy delivery duration of $2030 \pm 695 \mathrm{~s}$. No major adverse events were seen. In the cryoballoon group (Group B), a total of $133 \mathrm{PVs}$ were targeted using the $28-\mathrm{mm}$ balloon and $7 \mathrm{PVs}$ using the 23-mm balloon. Mean energy delivery duration was $3062 \pm 716$ s with a mean of $11 \pm 3$ applications. Of $140 \mathrm{PVs}, 128$ (91\%) were isolated using cryoballoon ablation alone, and $12(8 \%) \mathrm{PVs}$ were isolated using additional $3.5-\mathrm{mm}$ irrigated- 
Table 1. Patients' baseline characteristics in the four groups.

\begin{tabular}{lcccccc}
\hline & $\begin{array}{c}\text { All patients } \\
\text { (n = 110) }\end{array}$ & $\begin{array}{c}\text { Group 1 } \\
(\mathbf{n}=35)\end{array}$ & $\begin{array}{c}\text { Group 2 } \\
(\mathbf{n}=35)\end{array}$ & $\begin{array}{c}\text { Group 3 } \\
\text { (n= 20) }\end{array}$ & $\begin{array}{c}\text { Group 4 } \\
\text { (n = 20) }\end{array}$ & P \\
\hline Mean age & $57 \pm 11$ & $56 \pm 13$ & $58 \pm 12$ & $57 \pm 8$ & $58 \pm 10$ & 0.85 \\
Male & $84(76 \%)$ & $28(80 \%)$ & $23(66 \%)$ & $17(85 \%)$ & $16(80 \%)$ & 0.33 \\
AF history & $44 \pm 34$ & $47 \pm 41$ & $43 \pm 33$ & $50 \pm 30$ & $36 \pm 27$ & 0.57 \\
Lone AF & $58(53 \%)$ & $22(63 \%)$ & $17(49 \%)$ & $9(45 \%)$ & $10(50 \%)$ & 0.53 \\
Hypertension & $40(36 \%)$ & $12(34 \%)$ & $14(40 \%)$ & $8(40 \%)$ & $6(30 \%)$ & 0.86 \\
LA size [mm] & $42.1 \pm 5.4$ & $41.9 \pm 5.5$ & $41.5 \pm 5.1$ & $42.4 \pm 5.1$ & $43.2 \pm 6.4$ & 0.87 \\
EF [\%] & $61.9 \pm 7.3$ & $62.0 \pm 7.8$ & $61.8 \pm 7.1$ & $61.7 \pm 6.9$ & $62.3 \pm 7.4$ & 0.99 \\
CK [Ul/L] & $103 \pm 69$ & $111 \pm 74$ & $97 \pm 82$ & $105 \pm 56$ & $99 \pm 69$ & 0.97 \\
CK-MB [ng/mL] & $1.73 \pm 0.92$ & $1.64 \pm 0.78$ & $1.71 \pm 0.97$ & $1.89 \pm 1.04$ & $1.70 \pm 0.89$ & 0.93 \\
cTnl [ng/mL] & $0.03 \pm 0.01$ & $0.03 \pm 0.02$ & $0.02 \pm 0.01$ & $0.03 \pm 0.01$ & $0.03 \pm 0.01$ & 0.98 \\
\hline
\end{tabular}

Data are reported as mean \pm standard deviation; $A F-$ atrial fibrillation; $L A-$ left atrium; $E F$ - ejection fraction; CK - creatinine kinase; CK-MB — creatinine kinase-MB; cTnl — cardiac troponin I

Table 2. Procedural data in four different groups.

\begin{tabular}{lcccccc}
\hline & $\begin{array}{c}\text { All patients } \\
(\mathbf{n}=\mathbf{1 1 0})\end{array}$ & $\begin{array}{c}\text { Group 1 } \\
(\mathbf{n}=\mathbf{3 5})\end{array}$ & $\begin{array}{c}\text { Group 2 } \\
(\mathbf{n}=35)\end{array}$ & $\begin{array}{c}\text { Group 3 } \\
(\mathbf{n}=\mathbf{2 0})\end{array}$ & $\begin{array}{c}\text { Group 4 } \\
(\mathbf{n}=\mathbf{2 0})\end{array}$ & $\mathbf{P}$ \\
\hline Electroanatomic mapping & $79(72 \%)$ & $33(94 \%)$ & $10(29 \%)$ & $16(20 \%)$ & $20(100 \%)$ & $<0.0001$ \\
Intracardiac echocardiography & $32(29 \%)$ & $2(6 \%)$ & $6(17 \%)$ & $14(70 \%)$ & $10(50 \%)$ & $<0.0001$ \\
Double transseptal puncture & $37(34 \%)$ & $3(9 \%)$ & $13(37 \%)$ & $20(100 \%)$ & $1(5 \%)$ & $<0.0001$ \\
Mean energy delivery duration $[\mathrm{s}]$ & & $2030 \pm 695$ & $3062 \pm 716$ & $2511 \pm 1317$ & $2069 \pm 649$ & $<0.0001$ \\
\hline
\end{tabular}

tip RF ablation catheter in $10(28 \%)$ patients. No major complication occurred. In group $\mathrm{C}$ patients, there were a total of $78 \mathrm{PVs}$ and one left common trunk; all PVs and the common trunk were targeted and successfully isolated. The average laser energy delivery duration was $2511 \pm 1317 \mathrm{~s}$. All $\mathrm{PVs}$ remained isolated after a minimum of $30 \mathrm{~min}$ post-ablation. There was only a case of procedural pericardial effusions. In group D patients, there were a total of $76 \mathrm{PVs}$ and 2 left common trunks. All PVs isolation was obtained with a mean of $32 \pm 13 \mathrm{RF}$ applications and mean energy delivery duration of $2069 \pm 649 \mathrm{~s}$; the average contact force applied was $16 \pm 8 \mathrm{~g}$ (range 2-44 g) while mean total FTI was $37036 \pm 12527 \mathrm{~g}$ and mean FTI/RF application was $1371 \pm 1427 \mathrm{~g}$. No major complication occurred. Mean energy delivery duration differed significantly among four groups $(p=0.0001)$. In our series of 110 patients, $2(1.8 \%)$ patients complained chest discomfort $24 \mathrm{~h}$ post ablation ( 1 patients post RF ablation, 1 patients post cryoballoon). None of the patients had electrocardiographic or echocardiographic changes suggesting coronary ischemia.
Table 2 lists the procedural details in all patients and in the four different groups.

\section{Postprocedural cTnI and CK-MB levels}

The cTnI levels and CK-MB were normal in all patients pre-ablation and increased in all patients after the procedure. The first post procedural blood tests were performed at about $15 \pm 3 \mathrm{~h}$ after the beginning of RF ablation procedure. At the first postprocedural sample, cTnI levels were found elevated in all 110 patients with a median value of $2.11 \mathrm{ng} / \mathrm{mL}(0.20-19.85 \mathrm{ng} / \mathrm{mL})$. Patients treated with cryoablation showed levels of cTnI significantly increased (5.96; range 2.59-19.85 ng/ $\mathrm{mL}$ ) compared to the other groups (Group A: 1.72; range $0.22-5.73 \mathrm{ng} / \mathrm{mL}$; Group C: 1.54 ; range 0.20 $-4.13 \mathrm{ng} / \mathrm{mL}$; Group D: 2.0 ; range $1.0-7.81 \mathrm{ng} / \mathrm{mL}$ ) $(\mathrm{p}<0.001)$ (Table 3, Fig. 1). Among cryoablation group, in patients undergoing cryoablation with second generation cryo catheter, we found elevated levels of cTnI (median $5.01 \mathrm{ng} / \mathrm{mL}$ ), but we did not make any comparison because in this group we enrolled few patients. Also CK-MB levels were significantly elevated in 73 (66\%) patients; in particular 
Table 3. Postprocedural cardiac troponin I (cTnl) and creatinine kinase-MB (CK-MB) levels in four different groups.

\begin{tabular}{|c|c|c|c|c|c|c|}
\hline & $\begin{array}{l}\text { All patients } \\
\qquad(n=110)\end{array}$ & $\begin{array}{l}\text { Group } 1 \\
(n=35)\end{array}$ & $\begin{array}{l}\text { Group } 2 \\
(n=35)\end{array}$ & $\begin{array}{l}\text { Group } 3 \\
(n=20)\end{array}$ & $\begin{array}{l}\text { Group } 4 \\
(n=20)\end{array}$ & $\mathbf{P}$ \\
\hline cTnl 1 & $\begin{array}{c}2.11 \\
(0.20-19.85)\end{array}$ & $\begin{array}{c}1.72 \\
(0.22-5.73)\end{array}$ & $\begin{array}{c}5.96 \\
(2.59-19.85)\end{array}$ & $\begin{array}{c}1.54 \\
(0.20-4.13)\end{array}$ & $\begin{array}{c}2.0 \\
(1.0-7.81)\end{array}$ & $<0.0001$ \\
\hline cTnl 2 & $\begin{array}{c}0.96 \\
(0.07-10.06)\end{array}$ & $\begin{array}{c}0.80 \\
(0.08-2.94)\end{array}$ & $\begin{array}{c}2.46 \\
(0.72-10.06)\end{array}$ & $\begin{array}{c}0.69 \\
(0.07-1.45)\end{array}$ & $\begin{array}{c}0.84 \\
(0.21-2.75)\end{array}$ & $<0.0001$ \\
\hline CK-MB 1 & $\begin{array}{c}8.95 \\
(2.30-106.3)\end{array}$ & $\begin{array}{c}6.40 \\
(2.3-14.4)\end{array}$ & $\begin{array}{c}26.4 \\
(9.40-106.3)\end{array}$ & $\begin{array}{c}7.15 \\
(3.60-11.30)\end{array}$ & $\begin{array}{c}6.50 \\
(2.3-19.30)\end{array}$ & $<0.0001$ \\
\hline CK-MB 2 & $\begin{array}{c}3.2 \\
(0.90-54.0)\end{array}$ & $\begin{array}{c}2.1 \\
(0.90-11.5)\end{array}$ & $\begin{array}{c}6.4 \\
(1.89-54.0)\end{array}$ & $\begin{array}{c}2.7 \\
(1.6-4.2)\end{array}$ & $\begin{array}{c}2.2 \\
(1.1-4.9)\end{array}$ & $<0.0001$ \\
\hline
\end{tabular}

Data are reported as median and range. All values are in $\mathrm{ng} / \mathrm{mL}$; cTnl 1 and CK-MB 1 refer to first postprocedural samples; cTnl 2 and CK-MB 2 refer to second postprocedural samples.

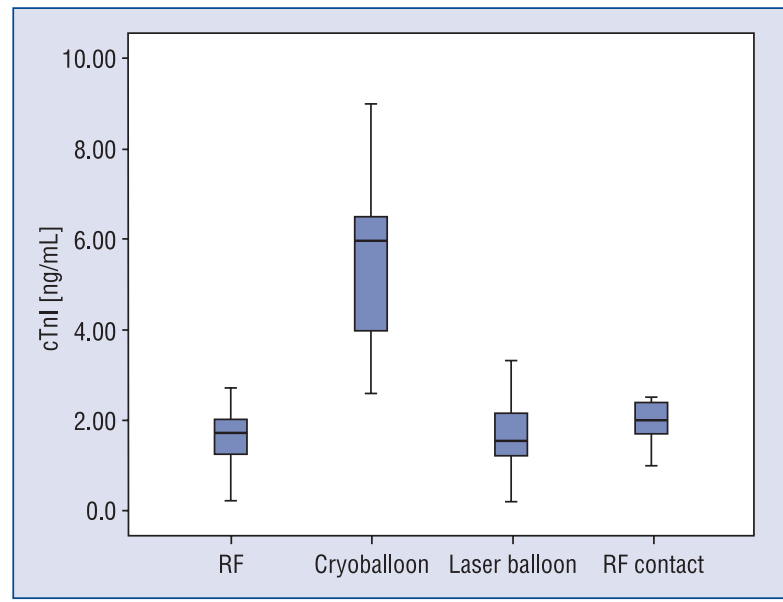

Figure 1. Cardiac troponin I (cTnl) median value at first postprocedural sample in four different groups; RF radiofrequency.

elevated CK-MB values were found in 17 out of 35 (49\%) Group A patients, in all 35 (100\%) Group B patients, in 11 out of 20 (55\%) Group C patients and in 10 out of $20(50 \%)$ Group D patients ( $<0.001)$. Patients treated with cryoablation showed significantly higher levels of CK-MB $(26.4 \mathrm{ng} / \mathrm{mL})$ compared to the others (Group A: $6.40 \mathrm{ng} / \mathrm{mL}$, Group C: $7.15 \mathrm{ng} / \mathrm{mL}$, and Group D: $6.50 \mathrm{ng} / \mathrm{mL}$ ) $(\mathrm{p}<0.001)$ (Table 3, Fig. 2).

Second post procedural blood tests were performed at about $40 \pm 3 \mathrm{~h}$ after the beginning of $\mathrm{RF}$ ablation procedure. c $\mathrm{TnI}$ levels were elevated in all 110 patients with a median value of 0.96 (range 0.07-10.06) ng/mL. Patients treated with cryoablation showed significantly higher levels of cTnI (2.46; range $0.72-10.06 \mathrm{ng} / \mathrm{mL}$ ) compared to patients of Group A (0.80; range $0.08-2.94 \mathrm{ng} / \mathrm{mL})$,

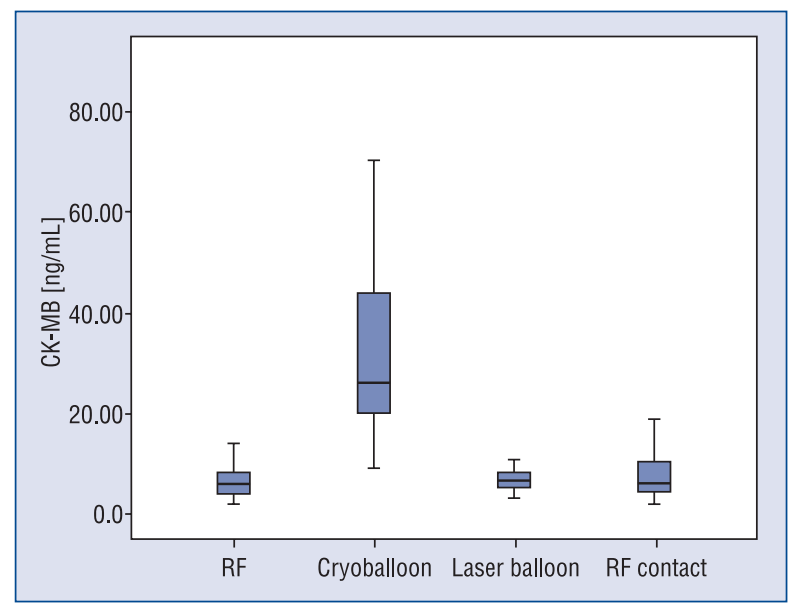

Figure 2. Creatinine kinase-MB (CK-MB) median value at first postprocedural sample in four different groups; $\mathrm{RF}$ - radiofrequency.

Group C (0.69; range 0.07-1.45 ng/mL), and Group D $(0.84$; range $0.21-2.75 \mathrm{ng} / \mathrm{mL})(\mathrm{p}<0.001)$ (Table 3).

Also CK-MB values were still above the level to detect myocardial injury in $19(17 \%)$ patients; in particular CK-MB values were found elevated in 16 (46\%) Group B patients, and in 3 (9\%) Group A patients. Patients treated with cryoablation showed significantly higher levels of CK-MB $(6.4 \mathrm{ng} / \mathrm{mL})$ compared to patients of Group A $(2.1 \mathrm{ng} / \mathrm{mL})$, Group C $(2.7 \mathrm{ng} / \mathrm{mL})$, and Group D $(2.48 \pm 1.07 \mathrm{ng} / \mathrm{mL})$ $(\mathrm{p}<0.001)$. Myocardial injury biomarker values are summarized in Table 3.

\section{Clinical outcomes after AF ablation}

After 1 year of follow-up, $22(20 \%)$ patients had clinical recurrences of $\mathrm{AF}, 7 / 35(20 \%)$ patients 


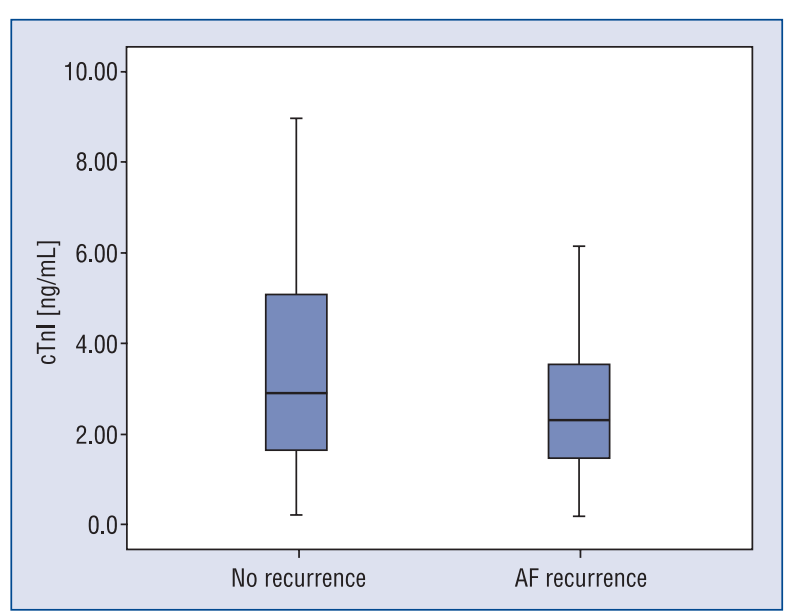

Figure 3. Cardiac troponin I (cTnl) median value at first postprocedural sample in two different groups: atrial fibrillation (AF) recurrences (27 patients) and no recurrences (83 patients).

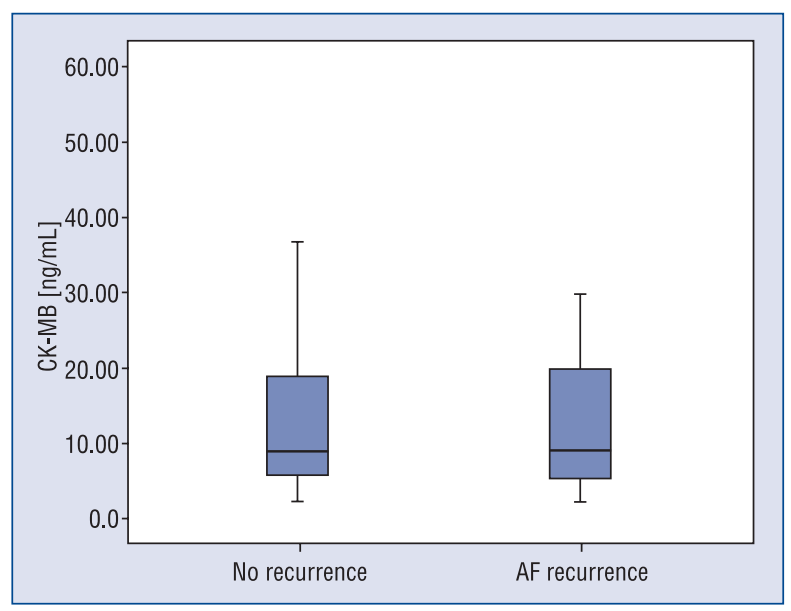

Figure 4. Creatinine kinase-MB (CK-MB) median value at first postprocedural sample in two different groups: atrial fibrillation (AF) recurrences (27 patients) and no recurrences (83 patients).

in Group A, 9/35 (25\%) in Group B, 3/20 (15\%) in Group C and 3/20 (15\%) in Group D. Because of the frequency of clinical symptoms, 10/27 (37\%) of these patients underwent repeat catheter ablation; $1(4 \%)$ patient underwent electrical cardioversion; the remaining $16 / 27$ (59\%) patients were successfully treated with a strengthened pharmacological therapy. Redo procedures were performed after a postprocedural blanking period of 3 months in 2 patients in the Group A, in 4 patients in the Group B, in 2 patients in the Group C and in 2 patients in the Group D ( $p=0.85)$.

\section{Analysis of biochemical markers with procedural and outcome data}

We also analyzed correlations of the levels of biochemical markers with the procedural data. There was a significant correlation between biomarker levels (first procedural sample) and longer energy delivery duration (for cTnI: $\mathrm{r}=0.90, \mathrm{p}=0.001$ and for CK-MB: $r=0.98, p=0.001$, respectively). The median value of cTnI and CK-MB (first post procedural sample) between $\mathrm{AF}$ recurrences group (22 patients) and no recurrences group (88 patients) was $2.1 \mathrm{ng} / \mathrm{mL}$ and $2.3 \mathrm{ng} / \mathrm{mL}$ for cTnI and $8.9 \mathrm{ng} / \mathrm{mL}$ and $9.1 \mathrm{ng} / \mathrm{mL}$, respectively (Figs. 3, 4). Since these median values were similar in the two groups, we did not perform any further analysis in order to assess an association.

\section{Discussion}

In our study, we compare 4 different ablation catheter technologies and energy sources for $\mathrm{AF}$ ablation with regard to their effect on myocardial necrosis. We performed AF ablation with one of the following technologies: irrigated tip RF catheter, cryoballoon, laser balloon, irrigated tip RF catheter with contact-force-sensing technology. Myocardial injury biomarkers were elevated in all patients undergoing PV isolation without any signs of cardiac ischemia by serial 12-lead electrocardiograms. Higher values of post-procedural cTnI and CK-MB were found in patients undergone cryoablation in comparison to patients treated with $\mathrm{RF}$ ablation or laser balloon. We also found a correlation between the levels of biomarkers and energy delivery duration, with higher values of energy delivery duration in cryoballoon group.

\section{Previous studies}

As several markers have been proven to be of clinical utility for diagnosis and estimation of infarct size, many authors tried to implement different biomarkers to evaluate and quantify the extent of effective ablation lesions. First studies focused on $\mathrm{RF}$ lesion and it has been demonstrated that cTnI and $\mathrm{cTnT}$ are more sensitive and specific than CK-MB for the diagnosis of myocardial injury after RF ablation $[5,6]$. It has also been showed that the rise of biomarkers and the extent of myocardial damage after RF catheter ablation depend on the number of $\mathrm{RF}$ pulses and site of ablation [12-16, 19]. A lower cTn level is expected after RF of focal area with few $\mathrm{RF}$ lesions. $\mathrm{RF}$ ablation of more myocardial tissue in the atria, such as in PV isolation, is expected to have much higher cTn levels [10-12, 20-25]. 
Previous clinical studies reported contrasting data, in the setting of $\mathrm{AF}$ ablation, on cardiac biomarkers levels after cryoballoon ablation compared to RF ablation [7, 13-19, 26]. Kuhne et al. [7] reported higher post procedural cTnT levels in RF group vs. cryoballoon group. Siklody et al. [10] analyzed hs-TnT and showed no significant difference in RF ablation and cryoballoon ablation. Only 1 study [6] reported increase of TnT levels in patients treated with cryoablation compared to those treated by RF ablation. Our finding that the cryoballoon resulted in a more pronounced myocardial injury is supported by 2 recent studies reporting higher cTnI levels after ablation with cryoballoon when comparing the PV ablation catheter using RF [19, 26]. In our study, the higher cTnI and CK-MB levels in cryoballoon group may be explained by longer energy delivery duration compared to the other groups. Previous AF ablation studies have found a correlation between levels of cTnT and RF delivery duration and cumulative RF energy [24, $27,28]$, but no data exist regarding other energy sources. Apart from the energy delivery duration, other factors may contribute to higher degree of myocardial injury in cryoablation, as suggested by increased levels of biomarkers post ablation. In fact, larger lesion can be obtained in both cryo and open irrigation $\mathrm{RF}$ ablation, depending on the conditions under which the lesions are created. Electrode orientation, contact pressure and blood velocity are the main factors involved in lesion formation. During cryoablation larger contact surface between the cryoballoon catheter and the endocardial tissue and the complete occlusion of the PV minimizing convective warming of the local blood velocity may create larger lesions [10, 28].

\section{Limitations of the study}

Only 2 measurements of biomarkers of myocardial damage levels were performed at $15 \pm 3 \mathrm{~h}$ and $43 \pm 3 \mathrm{~h}$ and no serial measurements were performed to assess a peak in TnI and CK-MB levels. Moreover, it was not possible to evaluate the kinetics of cTnI and CK-MB because of the lack of serial measurement. There are no published data about the release and kinetics of cTnI and CK-MB after catheter ablation with different energy sources so we cannot estabilish any comparison.

\section{Conclusions}

Compared to previous studies, we showed that cTnI and CK-MB are increased after PV isolation performed with 4 different ablation catheter technologies. Cardiac enzyme elevations are not specific for ischemia in the setting of chest pain after AF ablation. Higher values were found after cryoballoon ablation. It is possible that a longer delivery energy duration and other factors affecting lesion size resulted in higher amount of cardiac injury in cryoablation. However, the higher rise of cardiac biomarkers with cryoablation did not translate into a better clinical outcome and its physiologic significance is unknown.

Conflict of interest: C.T. has served as a member of the advisory board of Biosense Webster and has been a consultant for, and received lecture fees from, St. Jude Medical. A.N. has received compensation for belonging to the speakers' bureau for St. Jude Medical, Boston Scientific, Medtronic, and Biosense Webster and has received a research grant from St. Jude Medical. A.N. is also a consultant for Biosense Webster. L.D.B. is a consultant for Hansen Medical and Biosense Webster. The other authors declare no significant relationships with industry.

\section{References}

1. Haissaguerre M, Jais P, Shah DC et al. Spontaneous initiation of atrial fibrillation by ectopic beats originating in the pulmonary veins. N Engl J Med, 1998; 339: 659-666.

2. Mesas CE, Augello G, Lang CC et al. Electroanatomic remodeling of the left atrium in patients undergoing repeat pulmonary vein ablation: Mechanistic insights and implications for ablation. J Cardiovasc Electrophysiol, 2006; 17: 1279-1285.

3. Tang M, Kriatselis C, Nedios S et al. A novel cryoballoon technique for mapping and isolating pulmonary veins: A feasibility and efficacy study. J Cardiovasc Electrophysiol, 2010; 21: 626-631.

4. Reddy VY, Neuzil P, Themistoclakis S et al. Visually-guided balloon catheter ablation of atrial fibrillation: Experimental feasibility and first-in-human multicenter clinical outcome. Circulation, 2009; 120: 12-20.

5. Madrid AH, del Rey JM, Rubí J et al. Biochemical markers and cardiac troponin I release after radiofrequency catheter ablation: Approach to size of necrosis. Am Heart J, 1998; 136: 948-955.

6. Oswald H, Gardiwal A, Lissel C, Yu H, Klein G. Difference in humoral biomarkers for myocardial injury and inflammation in radiofrequency ablation versus cryoablation. Pacing Clin Electrophysiol, 2007; 30: 885-890.

7. Kühne M, Suter Y, Altmann D et al. Cryoballoon versus radiofrequency catheter ablation of paroxysmal atrial fibrillation: Biomarkers of myocardial injury, recurrence rates, and pulmonary vein reconnection patterns. Heart Rhythm, 2010; 7: 1770-1776.

8. Wojcik M, Janin S, Kuniss M et al. Limitations of biomarkers serum levels during pulmonary vein isolation. Rev Esp Cardiol, 2011; 64: 127-132.

9. Wójcik M, Janin S, Neumann T et al. Which standard biomarkers are useful for the evaluation of myocardial injury after pulmonary vein isolation with cryoballoon? Kardiol Pol, 2011; 69: 1151-1155. 
10. Siklody CH, Arentz T, Minners J et al. Cellular damage, platelet activation, and inflammatory response after pulmonary vein isolation: A randomized study comparing radiofrequency ablation with cryoablation Heart Rhythm, 2012; 9: 189-196.

11. Manolis AS, Vassilikos V, Maounis T et al. Detection of myocardial injury during radiofrequency catheter ablation by measuring serum cardiac troponin I levels: procedural correlates. J Am Coll Cardiol, 1999; 34: 1099-1105.

12. Alaiti AM, Maroo A, Edel TB. Troponin levels after cardiac electrophysiology procedures: review of the literature. Pacing Clin Electrophysiol, 2009; 32: 800-810.

13. Baust J. Cryotherapeutic intervention in cardiovascular disease (unpublished) 2002.

14. Budman H, Shitzer A, Dayan J. Analysis of the inverse problem of freezing and thawing of a binary solution during cryosurgical processes. J Biomech Eng, 1995; 117: 193-202.

15. Whittaker DK. Mechanisms of tissue destruction following cryosurgery. Ann R Coll Surg Engl, 1984; 66: 313-318.

16. Wood MA, Parvez B, Ellenbogen AL et al. Determinants of lesion sizes and tissue temperatures during catheter cryoablation. Pacing Clin Electrophysiol, 2007; 30: 644-654.

17. Parvez B, Pathak V, Schubert CM, Wood M. Comparison of lesion sizes produced by cryoablation and open irrigation radiofrequency ablation catheters. J Cardiovasc Electrophysiol, 2008; 19: 528-534.

18. Buttery JE, Stuart S, Pannall PR. Stability of the CK-MB isoenzyme on routine storage. Clin Biochem, 1992; 25: 11-13.

19. Schmidt M, Marschang $\mathrm{H}$, Clifford $\mathrm{S}$ et al. Trends in inflammatory biomarkers during atrial fibrillation ablation across different catheter ablation strategies. Int J Cardiol, 2012; 158: 33-38.

20. Haegeli LM, Kotschet E, Byrne J et al. Cardiac injury after percutaneous catheter ablation for atrial fibrillation. Europace, 2008; 10: $273-275$
21. Brueckmann M, Wolpert C Bertsch T et al. Markers of myocardial damage, tissue healing, and inflammation after radiofrequency catheter ablation of atrial tachyarrhythmias. J Cardiovasc Electrophysiol, 2004; 15: 696-691.

22. Manolis AS, Vassilikos V, Maounis T et al. Detection of myocardial injury during radiofrequency catheter ablation by measuring serum cardiac troponin I levels: procedural correlates. J Am Coll Cardiol, 1999; 34: 1099-1105.

23. Del Rey JM, Madrid AH, Valiño JM et al. Cardiac troponin I and minor cardiac damage: Biochemical markers in a clinical model of myocardial lesions. Clin Chem, 1998; 44: 2270-2276.

24. Carlsson J, Erdogan A, Guettler N et al. Myocardial injury during radiofrequency catheter ablation: comparison of focal and linear lesions. Pacing Clin Electrophysiol, 2001; 24: 962-968.

25. Katritsis DG, Hossein-Nia M, Anastasakis A et al. Myocardial injury induced by radiofrequency and low energy ablation: A quantitative study of $\mathrm{CK}$ isoforms, CK-MB, and troponin-T concentrations. Pacing Clin Electrophysiol, 1998; 21: 1410-1416 .

26. Malmborg H, Christersson C, Lönnerholm S, Blomström-Lundqvist C. Comparison of effects on coagulation and infla-mmatory markers using a duty-cycled bipolar and unipolar radiofrequency pulmonary vein ablation catheter vs. a cryoballoon catheter for pulmonary vein isolation. Europace, 2013; 15: 798-804.

27. Hirose H, Kato K, Suzuki O et al. Diagnostic accuracy of cardiac markers for myocardial damage after radiofrequency catheter ablation. J Interv Card Electrophysiol, 2006; 16: 169-174.

28. Emkanjoo Z, Mottadayen M, Givtaj N et al. Evaluation of post-radiofrequency myocardial injury by measuring cardiac troponin I levels. Int J Cardiol, 2007; 117: 173-177. 